\title{
Treatment Contrast Interaction
}

National Cancer Institute

\section{Source}

National Cancer Institute. Treatment Contrast Interaction. NCI Thesaurus. Code C142732.

The situation in which a treatment contrast (e.g., difference between investig ational product and control) is dependent on another factor (e.g., center). A quantitative interaction refers to the case where the magnitude of the contrast differs at the different levels of the factor, whereas for a qualitative interaction, the direction of the contrast differs for at least one level of the factor. (ICH) 